\title{
Phase transitions and thermodynamic properties of the antiferromagnetic Potts model on a face-centered cubic lattice
}

\author{
Albert Babaev ${ }^{1,2^{*}}$, Akai Murtazaev ${ }^{1,3}$, Felix Kassan-Ogly ${ }^{4}$, Alexey Proshkin ${ }^{4}$ \\ ${ }^{1}$ Institute of Physics of Daghestan Scientific Center of RAS, Makhachkala, Russia \\ ${ }^{2}$ Daghestan State Pedagogical University, Makhachkala, Russia \\ ${ }^{3}$ Daghestan State University, Makhachkala, Russia \\ ${ }^{4}$ Institute of Metal Physics, Ural Branch, RAS, Yekaterinburg, Russia
}

\begin{abstract}
Using the Monte Carlo method we investigate the phase transitions and thermodynamic properties of magnetic structures with noncollinear directions of magnetic moments corresponded to antiferromagnetic $q=4$ Potts model on a face-centered cubic lattice. Monte Carlo simulations are performed on lattices with linear sizes $L=20 \div 44$. Thermodynamic parameters: the order parameter $m_{A F}$, susceptibility $\chi$, internal energy $U$, and specific heat $C$ are evaluated for all studied systems. By employing the fourth order Binder cumulant method, a first order transition is shown to be occurred in the model.
\end{abstract}

\section{Introduction}

There exists at present a rich body of the experimental material on magnetic substances with crystalline structure and properties of $\mathrm{AnX}$-type compounds with the $\mathrm{NaCl}$ structure. These are so called monopnictides and monochalcogenides, where $A n$ is a rear-earth element or actinide, $X$ is V or VI groups' element (UN, UAs, USb). They display most interesting properties. The evolution of the magnetic neuronography increases a number of experiments in determination of different compound magnetic structures. Rossat-Mignod et. al. [1] proved that a single wave vector component is insufficient to describe the experimental data for $U P, U A s$, and $U S b$ compounds. Therefore, multi-k structures with wave vectors comprised more than one component (2-k structures for two-component wave vector and 3-k structures for three-component vector) were proposed. The interpretation of $U S b$ neuronography is in a better agreement with experimental data of a 3-k structure than a collinear model. We notice that this $3-\mathbf{k}$ structure corresponds precisely to the $q=4$ standard Potts model illustrated in Fig. 1.

The investigation of phase transitions (PTs) and thermodynamic properties of the 4-state antiferromagnetic (AF) Potts model is the main goal of this study.

\footnotetext{
*Corresponding author: b_albert78@mail.ru
} 


\section{Model and Method}

The Hamiltonian of the antiferromagnetic $q=4$ standard Potts model on a face-centered cubic (FCC) lattice (Fig.1) reads

$$
H=-J \sum_{i, j} \cos \theta_{i, j}
$$

where $J$ is the parameter of antiferromagnetic exchange interaction $(J<0), \theta_{i, j}$ is the angle between interacting spins $S_{i}-S_{j}$. As is evident from Fig. 1, in the model spins occupying lattice sites can orient in hypertetrahedron $q$-symmetric directions in the dimensionality space $q-1$ retaining equal angles between any two different directions.

The cubic ferromagnetic $q=4$ Potts model with nearest neighbor ferromagnetic interactions were studied in works $[2,3]$. Authors reported that a second order transition occurred in the models considered.
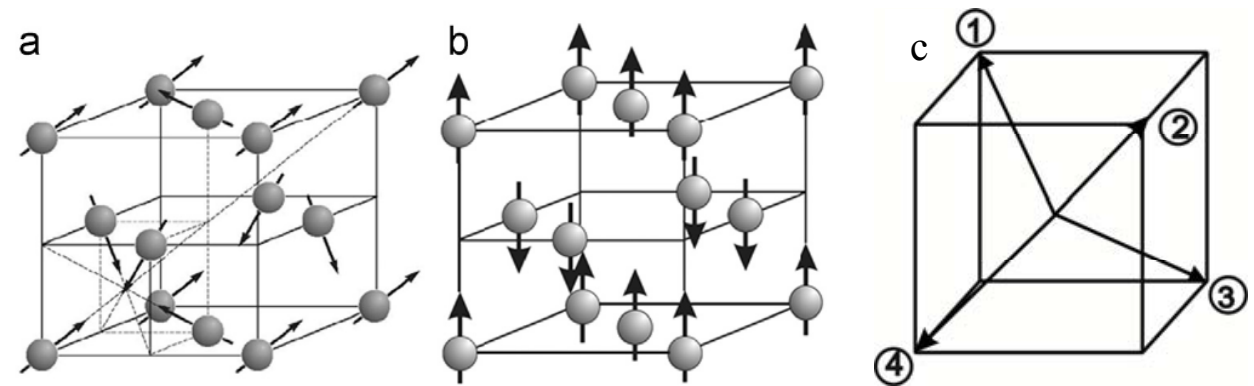

Fig. 1. Identification of the USb diffraction pattern in the $3-k$ structure (a) and in the collinear one (b). 4-State Potts model (c).

In our study we use the Metropolis algorithm along with the Wolff cluster algorithm [4]. We performe the Monte Carlo simulations for systems of linear sizes $4 \times(L \times L \times L)$ with periodic boundary conditions, where $L=28-40$. Initial configurations were given so that every spin inside each of sublattices is ordered. To bring the system to equilibrium state the relaxation time $\tau_{0}$ for all systems with $L$ is computed. Next, the averaging is performed along the Markov chain of length $\tau=150 \tau_{0}$ and additional averaging is done by ten different initial spin configurations.

\section{Simulation Results}

The temperature behavior of the specific heat and susceptibility is evaluated from fluctuation correlations [5]

$$
\begin{aligned}
& C=\left(N K^{2}\right)\left(\left\langle U^{2}\right\rangle-\langle U\rangle^{2}\right), \\
& \chi=(N K)\left(\left\langle m^{2}\right\rangle-\langle m\rangle^{2}\right),
\end{aligned}
$$

where $K=|J| / k_{B} T, N=4 \times L^{3}$ is the number of sites, $U$ is the intrinsic energy, $m$ is the order parameter, angle brackets denotes the ensemble average. The order parameter for the antiferromagnetic Potts model is defined as [6]

$$
m_{A F}=\left\langle\frac{4}{3} \sum_{\alpha \neq \beta \neq \gamma \neq \varphi}\left(\frac{\left(N_{\alpha}+N_{\beta}+N_{\gamma}+N_{\varphi}\right.}{N}-\frac{1}{4}\right)\right\rangle^{1 / 2},
$$


where $N_{\alpha}=\left\{N_{1}, N_{2}, N_{3}, N_{4}\right\}, N_{1}, N_{2}, N_{3}$, and $N_{4}$ are the number of spins in $q=1, q=2, q=3$, and $q=4$, correspondingly, $N_{\alpha}, N_{\beta}, N_{\gamma}, N_{\varphi}$ are the number of spins in sublattices $A, B, C$, and $D$, correspondingly.

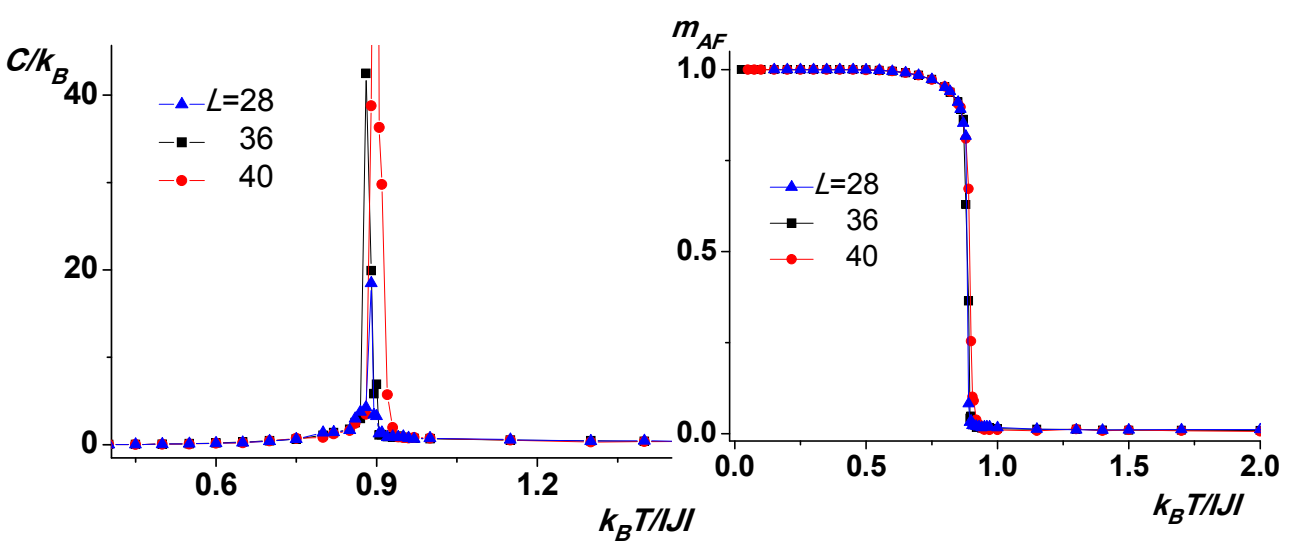

Fig. 2. The temperature dependence of the specific heat Fig. 3. The temperature dependence of the $C$ for the 3D 4-state Potts model on the FCC lattice. order parameter $m_{A F}$ for the 3D 4-state Potts model on the FCC lattice.

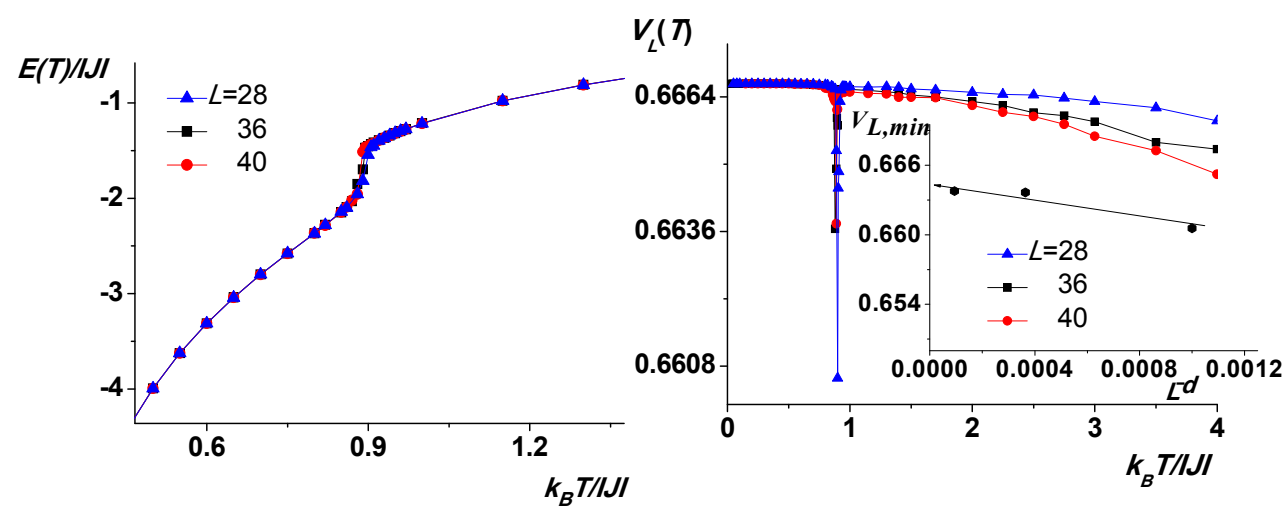

Fig. 4. The temperature dependence of the average energy for the 3D 4-state Potts model on the FCC lattice.
Fig. 5. Dependence of the Binder cumulants $V_{L}(T)$ on temperature for the $3 \mathrm{D} 4$-state Potts model on the FCC lattice.

Figures 2, 3, 4 and 5 depict the temperature dependences of the specific heat $C$, order parameter $m_{A F}$, internal energy $E$ and Binder cumulants $V_{L}$ for 3D 4-state antiferromagnetic Potts model on a FCC lattice for systems with $L=28 ; 36 ; 40$. From now on an error of data in all figures doesn't exceed symbol sizes used at plotting the diagrams. As is evident from Figs. 2, 3, and 4, the temperature dependences of the specific heat $C$, order parameter $m_{A F}$, internal energy $E$ for all studied systems exhibit a behavior featured to a first order transition (a jump occurs at PT point). Beyond that, we investigate the dependence of the Binder's cumulants $V_{L}(T)$ on temperature $T$ for this model (Fig. 5). A clearly marked jump at PT point indicates a first order transition. The fourth-order Binder cumulants method [7] has proved to be the most efficient method for the analysis of the character of the phase transition at $T=T_{t}(L)$. 


$$
\begin{gathered}
V_{L}(T)=1-\frac{\left\langle E^{4}\right\rangle_{L},}{3\left\langle E^{2}\right\rangle_{L}^{2}} \\
U_{L}(T)=1-\frac{\left\langle m^{4}(T, L)\right\rangle_{L}}{3\left\langle m^{2}(T, L)\right\rangle_{L}{ }^{2}},
\end{gathered}
$$

where $E$ is the internal energy and $m$ is the order parameter of the system with $L$. This technique has proved to be successful for the detection of a PT order, a detail description of that was provided in [8-12]. The averaged value $V_{L}(T)$ tends to a certain non-trivial value $V^{*}$ at the first order transition

$$
V(T)=V^{*}+b L^{-d}
$$

at $L \rightarrow \infty$ and $T=T_{t}(L)$, where $V^{*}$ is distinct from $2 / 3$ at $L \rightarrow \infty$, which is shown in the insert in Fig. 5 for the 3D $q=4$ antiferromagnetic Potts model on a FCC lattice.

Thus, in this study we evaluate within the unified technique the phase transitions and thermodynamic properties of magnetic structures with non-collinear directions of magnetic moments corresponded to the antiferromagnetic $q=4$ Potts model on a FCC lattice. The temperature dependences of thermodynamic parameters for the specific heat $C$, susceptibility $\chi$, order parameter $m_{A F}$, and Binder cumulants $V_{L}(T)$ demonstrate the first order transition. The investigation of the antiferromagnetic $q=4$ Potts model on a FCC lattice permits to elucidate magnetic and thermodynamic properties of real crystals with multi- $\mathbf{k}$ structures.

\section{References}

1. J. Rossat-Mignod, Neutron scattering. In: Methods of experimental physics (New York: Academic Press, 1987)

2. A.K. Murtazaev, A.B. Babaev, G.Y. Aznaurova, Solid State Phenomena 168-169, 357 (2011)

3. A.K. Murtazaev, A.B. Babaev, G.Y. Aznaurova, Low Temperature Physics 37, 134 (2011)

4. U. Wolff. Phys. Lett. 62, 361 (1989)

5. P. Peczac, A.M. Ferrenberg, D.P. Landau, Phys.Rev. B 43, 6087 (1991)

6. Y. Saito, J. Phys. A 15, 1885, (1982)

7. K. Eichhorn, K. Binder, J. Phys.: Condens. Matter 8, 5209 (1996)

8. A.K. Murtazaev, A.B. Babaev, JETP Lett. 99, 535 (2014)

9. A.B. Babaev, A.K. Murtazaev, Low temperature physics 41, 608 (2015)

10. A.K. Murtazaev, A.B. Babaev, G.Ya. Ataeva, Low Temperature Physics 39, 147 (2013)

11. A.K. Murtazaev, A.B. Babaev, G. Ya. Ataeva, Physics of the solid state 59, 141 (2017)

12. A.B. Babaev, T.R. Rizvanova, A.K. Murtazaev, Physics of the solid state 59, 2444 (2017) 